\title{
Can we use baseline characteristics to assess which men with moderately symptomatic benign prostatic hyperplasia at risk of progression will benefit from treatment? A post hoc analysis of data from the 2-year CONDUCT study
}

\author{
Claus G. Roehrborn ${ }^{1}$ Igor Oyarzabal Perez ${ }^{2} \cdot$ Erik P. M. Roos ${ }^{3} \cdot$ \\ Nicolae Calomfirescu ${ }^{4} \cdot$ Betsy Brotherton $^{5,8} \cdot$ Juan Manuel Palacios $^{6} \cdot$ \\ Averyan Vasylyev $^{7} \cdot$ Michael J. Manyak $^{5}$ \\ Received: 20 April 2016 / Accepted: 15 June 2016 / Published online: 22 June 2016 \\ (C) The Author(s) 2016. This article is published with open access at Springerlink.com
}

\begin{abstract}
Purpose To investigate (in a post hoc analysis of the 2-year CONDUCT study) the characteristics and clinical outcomes of men with moderately symptomatic benign prostatic hyperplasia (BPH) at risk of progression who benefitted from lifestyle changes alone.

Methods Patients were given lifestyle advice and randomized to a fixed-dose combination (FDC) of dutasteride and tamsulosin or watchful waiting (WW) and followed for 24 months. Patients in the WW group were escalated to tamsulosin if any follow-up International Prostate Symptom Score (IPSS) was equal or greater than the baseline value. Improvements in symptoms (change in IPSS) and quality of life [measured by BPH Impact Index (BII) and question 8 of the IPSS (IPSS-Q8)] were analysed in the FDC group, men who initiated tamsulosin (WW-TAM) and men who
\end{abstract}

Claus G. Roehrborn

Claus.roehrborn@utsouthwestern.edu

1 Department of Urology, UT Southwestern Medical Center, 5323 Harry Hines Blvd, J8 142, Dallas, TX 75390-9110, USA

2 Department of Urology, Mendaro Hospital, Mendaro, Gipuzkoa, Spain

3 Department of Urology, Antonius Hospital Sneek, Sneek, The Netherlands

4 Urology Clinic, Uroandromed, Bucharest, Romania

5 GlaxoSmithKline, Research Triangle Park, NC, USA

6 GlaxoSmithKline, Urology, Classic and Established Products, GlaxoSmithKline, Madrid, Spain

7 GlaxoSmithKline Pharmaceuticals, GlaxoSmithKline, Kiev, Ukraine

8 Present Address: PAREXEL International, Durham, NC, USA received no medical intervention (WW-no treatment) and the impact of baseline variables on IPSS determined.

Results The adjusted mean decrease in IPSS, BII and IPSSQ8 at each post-baseline visit over 24 months appeared greater in the FDC $(n=369)$ and WW-no treatment groups $(n=144)$ than in the WW-TAM group $(n=229)$. IPSS improvements appeared similar in the FDC group and WW-no treatment subgroup, except in patients with the greatest degree of bother at baseline (BII 7-13).

Conclusion BII at baseline may be a more relevant indicator than symptom severity as to whether a patient with moderate symptoms should receive medical therapy or not.

Keywords Benign prostatic hyperplasia $\cdot$ BPH Impact Index $\cdot$ Dutasteride $\cdot$ Lower urinary tract symptoms ·

Tamsulosin $\cdot$ Watchful waiting

$\begin{array}{ll}\text { Abbreviations } \\ \text { BII } & \text { BPH Impact Index } \\ \text { BPE } & \text { Benign prostatic enlargement } \\ \text { BPH } & \text { Benign prostatic hyperplasia } \\ \text { FDC } & \text { Fixed-dose combination } \\ \text { IPSS } & \text { International Prostate Symptom Score } \\ \text { LUTS } & \text { Lower urinary tract symptoms } \\ \text { PSA } & \text { Prostate-specific antigen } \\ \text { QoL } & \text { Quality of life } \\ \text { WW } & \text { Watchful waiting }\end{array}$

\section{Introduction}

More than $50 \%$ of men aged $\geq 60$ years have histological evidence of benign prostatic hyperplasia (BPH). BPH can be associated with benign prostatic enlargement (BPE), which can result in lower urinary tract symptoms (LUTS) 
such as weak stream and hesitancy; some men may also experience LUTS associated with storage, such as increased urgency, frequency and nocturia. These symptoms can significantly diminish a person's quality of life (QoL) $[1,2]$.

The optimal time point to initiate medical therapy of BPH is a matter of debate [3]. Although guidelines recommend a conservative approach for men with minimal symptoms $[4,5]$, several observations argue that early intervention may be an appropriate option for some patients $[6,7]$.

The CONDUCT study was performed to investigate whether there was a difference in symptomatic improvement from baseline, as measured by the International Prostate Symptom Score (IPSS), among treatment-naïve men with moderately symptomatic BPH at risk of progression, who were given lifestyle advice and either immediate treatment with a fixed-dose combination (FDC) of dutasteride and tamsulosin or managed with watchful waiting (WW) with protocol-defined initiation of tamsulosin monotherapy if symptoms did not improve. The results of this study have previously been reported [8]. Notably, over one-third of men managed with WW in CONDUCT had symptomatic improvement without any pharmacological intervention, i.e. with lifestyle advice alone [8].

The mechanisms leading to an improvement in symptoms without pharmacologic intervention are poorly understood; however, condition-, patient- and lifestyle-specific factors could be involved [3]. To investigate this further, we report a post hoc analysis of CONDUCT 2-year data revealing the demographics, disposition and clinical outcomes of men who received immediate intervention with FDC, initiated tamsulosin monotherapy or received no pharmacological intervention and benefitted from lifestyle advice alone.

\section{Patients and methods}

\section{Study design}

The design of the CONDUCT study (GlaxoSmithKline FDC114615; NCT01294592) has been reported [8]. Eligible men [aged $\geq 50$ years, confirmed clinical diagnosis of BPH, moderate LUTS (IPSS of 8-19), prostate volume $\geq 30 \mathrm{~mL}$ and total serum prostate-specific antigen (PSA) level of $\geq 1.5 \mathrm{ng} / \mathrm{mL}$ ] were randomized $1: 1$ to self-administer a FDC of dutasteride $0.5 \mathrm{mg}$ and tamsulosin $0.4 \mathrm{mg}$ QD (Duodart ${ }^{\circledR}$, GlaxoSmithKline), or WW with initiation of tamsulosin $0.4 \mathrm{mg}$ QD if any IPSS after randomization was the same or greater than the baseline value. Lifestyle advice was provided at baseline to all patients.

Patients visited the clinic 4 weeks after randomization and at 13-week intervals thereafter, for up to 24 months. Patient-reported symptoms, symptom impact and
BPH-related QoL were assessed at every visit using the last observation carried forward method. Data on adverse events were collected from first administration of study treatment (FDC or tamsulosin) until discontinuation and have been presented previously [8]. Safety data were not scheduled to be collected for patients in the WW group who received no drug.

\section{Post hoc data analyses}

Symptomatic improvement, as measured by IPSS, was investigated in the FDC group, the WW subgroup who initiated tamsulosin (WW-TAM) and the WW subgroup who did not receive medical intervention (WW-no treatment). As the CONDUCT study was not powered to assess treatment effects in or across the WW-TAM and WW-no treatment subgroups, no formal statistical analyses were performed. Data from patients in the WW-TAM and WW-no treatment subgroups were supplemental and are provided for descriptive purposes only.

Data were summarised overall and by baseline subgroups as tabular displays and figures in terms of mean change from baseline in IPSS, BPH Impact Index (BII) and question 8 of the IPSS (IPSS-Q8). Changes in IPSS were also summarised according to the categorical subgroups of age $(<65, \geq 65$ years) and BII $(0-3,4-6$ or $7-13$ at baseline).

\section{Results}

\section{Patient demographics and disposition}

In all, 742 patients were randomized into CONDUCT (369 and 373 in the FDC and WW groups, respectively). Of the 373 patients randomized to WW, $229(61.4 \%)$ initiated tamsulosin, mostly within the first 6 months of the study. Of 190 patients who started tamsulosin within 6 months of randomization, 125 (66\%) started in month 1, $45(24 \%)$ in month 3 and $20(11 \%)$ in month 6 . Baseline characteristics were generally similar between the three groups (Table 1). Although the mean IPSS at baseline was the same for men in the WW-TAM and WW-no treatment subgroups, men in the WW-no treatment subgroup tended to be younger and less bothered by their symptoms at baseline.

\section{Changes in IPSS, BII and IPSS-Q8}

The adjusted mean decrease (improvement) in IPSS at each post-baseline visit over 24 months appeared greater in the WW-no treatment subgroup than in the WW-TAM subgroup and very similar between the FDC group and WW-no treatment subgroup (Fig. 1a). At month 24, the 
Table 1 Baseline characteristics

\begin{tabular}{lllll}
\hline Variable & FDC $(n=369)$ & $\begin{array}{l}\text { WW-TAM } \\
(n=229)\end{array}$ & $\begin{array}{l}\text { WW-no treatment } \\
(n=144)\end{array}$ & All $(N=742)$ \\
\hline Age (years) & 66.3 & 66.7 & 65.3 & 66.2 \\
PSA (ng/mL) & 3.9 & 3.8 & 3.6 & 3.8 \\
Prostate volume (mL) & 51.0 & 52.6 & 52.6 & 51.8 \\
Mean IPSS & 13.2 & 12.9 & 12.9 & 13.1 \\
Mean BII score & 4.8 & 4.7 & 4.0 & 4.6 \\
BII 0-3 $[n(\%)]$ & $123(33)$ & $75(33)$ & $68(47)$ & $266(36)$ \\
BII 4-6 $[n(\%)]$ & $132(36)$ & $94(41)$ & $37(26)$ & $263(35)$ \\
BII 7-13 $[n(\%)]$ & $114(31)$ & $60(26)$ & $39(27)$ & $213(29)$ \\
IPSS-Q8 & 3.2 & 3.1 & 2.9 & 3.1 \\
\hline
\end{tabular}
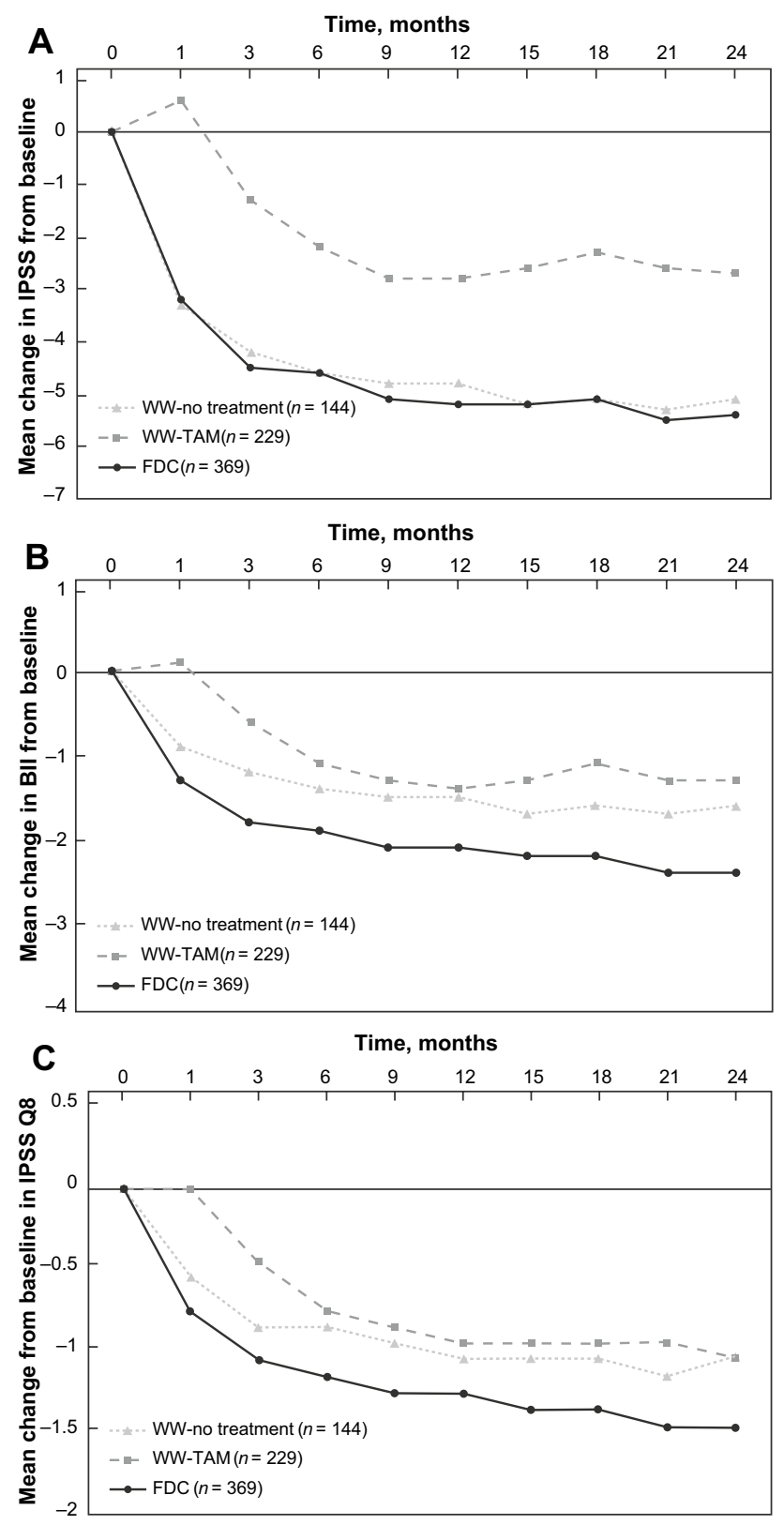

Fig. 1 Mean change from baseline in a IPSS, b BII and c IPSS-Q8 mean change in IPSS was -5.6 for patients in the FDC group, -5.1 for patients in the WW-no treatment subgroup and -2.7 among patients who received tamsulosin.

The improvement in BII and IPSS-Q8 at each postbaseline visit over 24 months appeared greater in the FDC group than in either the WW-no treatment or the WW-TAM subgroups and greater in the WW-no treatment subgroup than in the WW-TAM subgroup (Fig. 1b, c).

\section{Impact of baseline variables on changes in IPSS}

The baseline characteristics of men aged $\geq 65$ or $<65$ years of age were generally comparable (Table 2). Although men aged $<65$ years were more bothered by their urinary problems at baseline than older men (BII score of 5.1 vs 4.3), this did not translate into a difference in baseline IPSS. In the FDC and WW-no treatment subgroups, the mean IPSS was lower at each post-baseline visit in patients aged $<65$ years than in patients aged $\geq 65$ years. Between baseline and month 24, the IPSS for patients aged $<65$ and $\geq 65$ years improved by 6.6 and 4.8 points for patients in the FDC group, 5.5 and 4.8 points for patients in the WW-no treatment subgroup and 2.9 and 2.6 points in the WW-TAM subgroup (Fig. 2a; Table 3).

Table 3 shows the mean IPSS change from baseline at 24 months according to various categories of baseline characteristics. Symptom improvement in the WW-no treatment subgroup was similar across the different categories, with no obvious signals of a baseline characteristic that indicated better symptom outcomes. IPSS improvements generally appeared greater in the FDC group and WW-no treatment subgroup than in the WW-TAM subgroup and were similar in the FDC group and WW-no treatment subgroups, except in patients with the greatest degree of bother at baseline. Between baseline and month 24, the IPSS for patients with baseline BII of 0-3, 4-6 and 7-13 improved by 4.3, 5.4 and 7.1 points for patients in the FDC group, 5.0, 5.3 and 5.1 points for patients in the WW-no treatment subgroup and 1.6, 2.7 and 4.1 points in the WW-TAM subgroup (Fig. 2b). 
Table 2 Baseline characteristics of patients according to age

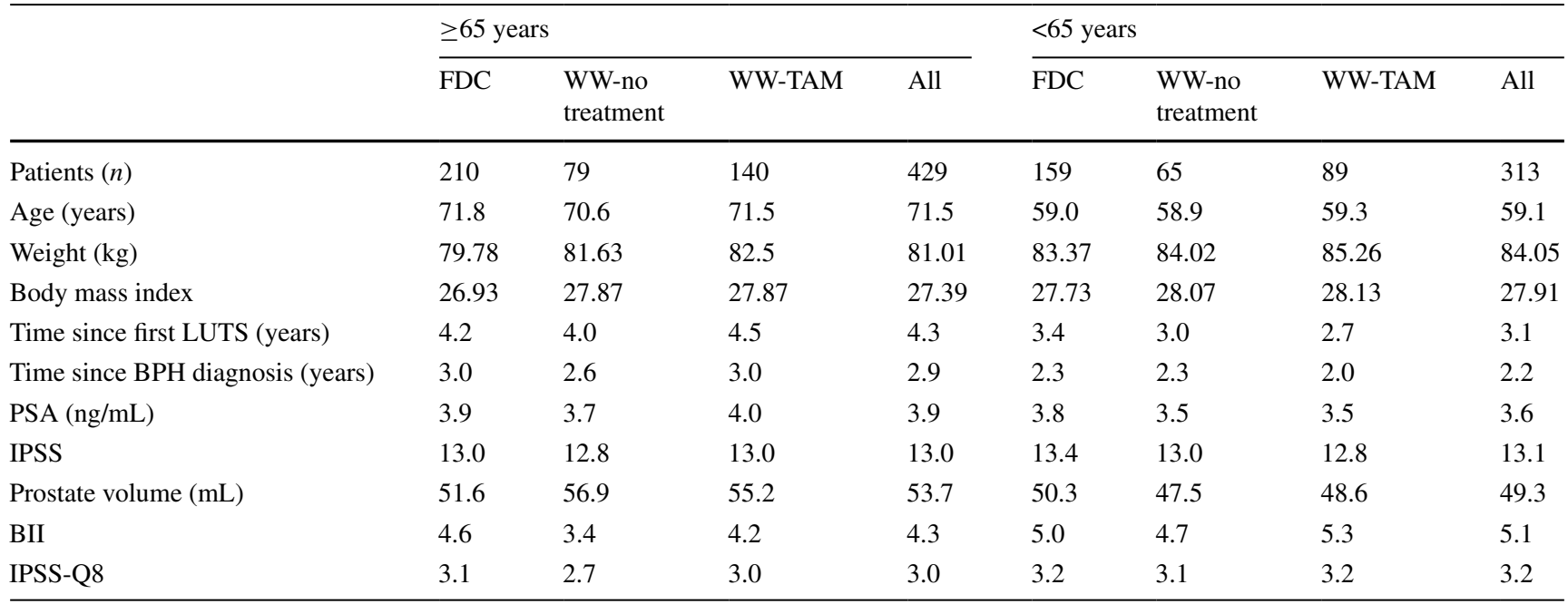

Fig. 2 Mean change from baseline in IPSS according to a age (solid line $\geq 65$ years; broken line $<65$ years) and $\mathbf{b}$ BII at baseline

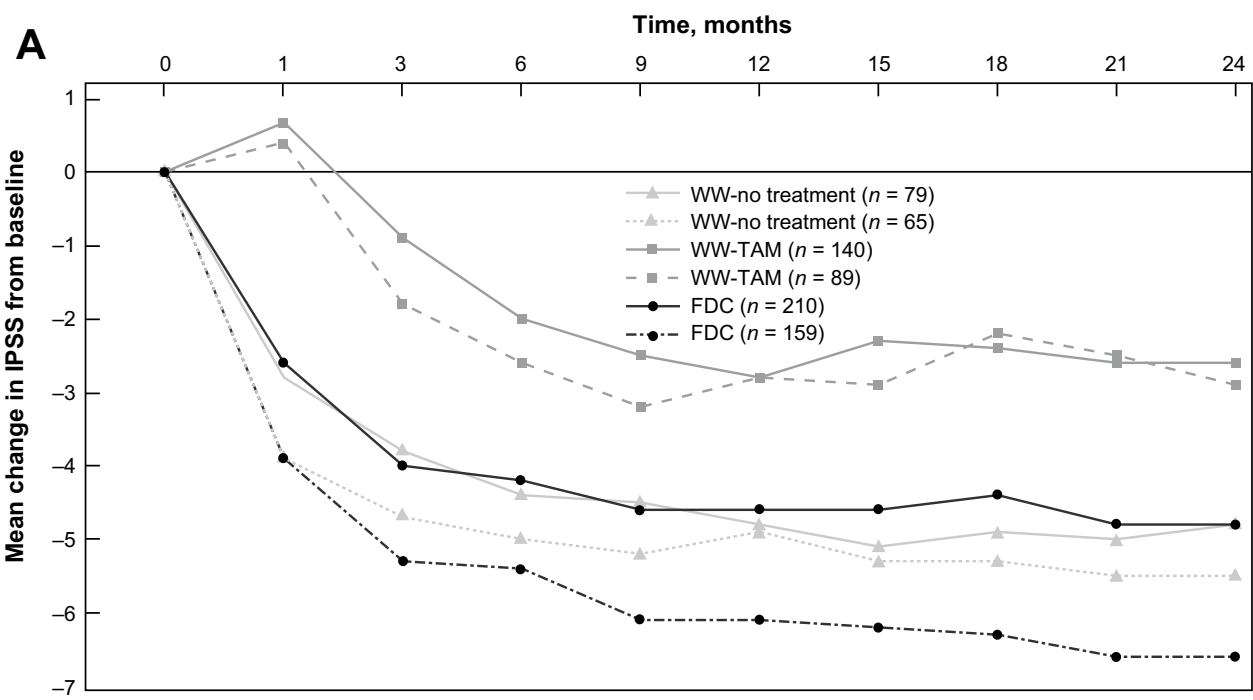

Time, months

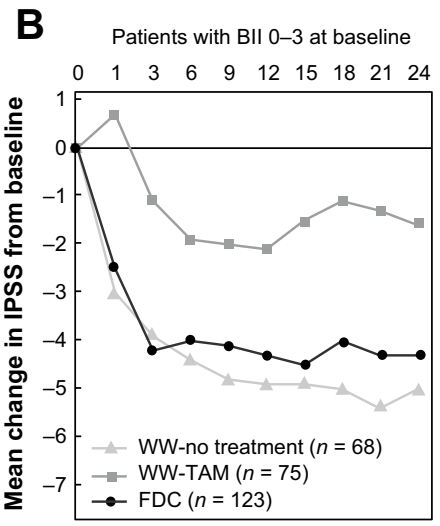

Patients with BII 4-6 at baseline

Patients with BII 7-13 at baseline
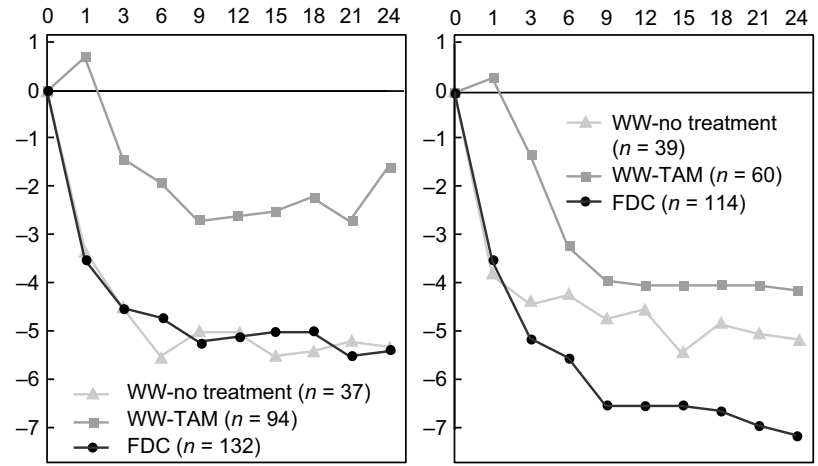
Table 3 Mean IPSS change from baseline at 24 months according to baseline characteristics

\begin{tabular}{|c|c|c|c|c|c|c|c|c|}
\hline \multirow[t]{3}{*}{ Baseline characteristic } & \multicolumn{8}{|c|}{ Mean change in IPSS from baseline at month 24 (patients, $n$ ) } \\
\hline & \multicolumn{4}{|c|}{ Patients aged $\geq 65$ years at baseline } & \multicolumn{4}{|c|}{ Patients aged $<65$ years at baseline } \\
\hline & FDC & WW-All & $\begin{array}{l}\text { WW-no } \\
\text { treatment }\end{array}$ & WW-TAM & FDC & WW-All & $\begin{array}{l}\text { WW-no } \\
\text { treatment }\end{array}$ & WW-TAM \\
\hline$\geq$ or $<65$ years & $-4.8(203)$ & $-3.3(216)$ & $-4.8(76)$ & $-2.6(140)$ & $-6.6(156)$ & $-4.0(152)$ & $-5.5(63)$ & $-2.9(89)$ \\
\hline IPSS $<13$ & $-3.3(92)$ & $-1.7(108)$ & $-3.9(42)$ & $-0.4(66)$ & $-4.7(77)$ & $-3.4(69)$ & $-5.1(31)$ & $-2.0(38)$ \\
\hline IPSS $\geq 13$ & $-5.9(111)$ & $-4.9(108)$ & $-5.9(34)$ & $-4.5(74)$ & $-8.5(79)$ & $-4.5(83)$ & $-5.9(32)$ & $-3.7(51)$ \\
\hline BII 0-3 & $-4.1(69)$ & $-2.7(98)$ & $-4.4(43)$ & $-1.4(55)$ & $-4.6(49)$ & $-4.2(43)$ & $-6.1(23)$ & $-2.0(20)$ \\
\hline BII 4-6 & $-4.5(78)$ & $-3.7(77)$ & $-5.9(20)$ & $-3.0(57)$ & $-6.9(52)$ & $-2.9(53)$ & $-4.5(16)$ & $-2.2(37)$ \\
\hline BII 7-13 & $-5.9(56)$ & $-4.0(41)$ & $-4.3(13)$ & $-3.9(28)$ & $-8.3(55)$ & $-4.9(56)$ & $-5.6(24)$ & $-4.3(32)$ \\
\hline IPSS Q8 <4 & $-4.4(133)$ & $-3.0(146)$ & $-4.7(54)$ & $-1.9(92)$ & $-5.4(96)$ & $-4.0(96)$ & $-5.4(40)$ & $-2.9(56)$ \\
\hline IPSS Q8 $\geq 4$ & $-5.4(70)$ & $-4.1(70)$ & $-4.9(22)$ & $-3.7(48)$ & $-8.7(60)$ & $-4.1(56)$ & $-5.7(23)$ & $-3.0(33)$ \\
\hline Prostate volume $<40 \mathrm{~mL}$ & $-4.7(67)$ & $-3.6(49)$ & $-4.7(17)$ & $-3.0(32)$ & $-5.9(54)$ & $-4.2(47)$ & $-5.3(21)$ & $-3.3(26)$ \\
\hline Prostate volume $\geq 40 \mathrm{~mL}$ & $-4.8(135)$ & $-3.3(167)$ & $-4.8(59)$ & $-2.4(108)$ & $-7.0(102)$ & $-3.9(105)$ & $-5.6(42)$ & $-2.8(63)$ \\
\hline $\mathrm{PSA}<3 \mathrm{ng} / \mathrm{mL}$ & $-3.8(78)$ & $-4.2(82)$ & $-5.1(31)$ & $-3.6(51)$ & $-6.2(69)$ & $-4.3(77)$ & $-6.0(31)$ & $-3.1(46)$ \\
\hline $\mathrm{PSA} \geq 3 \mathrm{ng} / \mathrm{mL}$ & $-5.3(125)$ & $-2.8(134)$ & $-4.6(45)$ & $-1.9(89)$ & $-7.0(87)$ & $-3.7(75)$ & $-5.0(32)$ & $-2.8(43)$ \\
\hline
\end{tabular}

\section{Discussion}

Symptomatic improvements among men in the WW-no treatment subgroup appeared similar to those in men who received FDC therapy and better than those in the WWTAM subgroup. There are a number of possible explanations for this. As previously reported [8], men who initiated tamsulosin tended to be older and have more bothersome symptoms at baseline. It is possible that younger men are better able to tolerate their symptoms; as a result, the degree of bother does not translate into changes in IPSS and treatment with tamsulosin is not indicated. By contrast, the threshold between the degree of bother and impact on IPSS may be lower in elderly men; i.e. although they may not have required medical intervention, the impact of symptoms on their general well-being may have led them to actively seek treatment. Another possible explanation relates to the study design. The WW-no treatment subgroup is composed of men who, from one study visit to the next, exhibited a lower score compared with baseline. CONDUCT selected a group of men in this arm who, perhaps by random choice, had a baseline symptom score higher than their true symptom status before enrolment. Over the course of the study, they never reached the high score recorded at baseline. Since all men whose symptom score stayed the same or increased were eliminated from this subgroup; this may have resulted in a remarkable (albeit artificial) 'improvement' that cannot be attributed to a placebo effect.

In the present post hoc analysis, symptom improvement was analysed according to age and BII at baseline. The data show that in terms of active treatment, FDC therapy appeared more effective than tamsulosin monotherapy in improving symptoms and lessening the degree of associated bother across all baseline subgroups. FDC therapy also offered an advantage over no treatment in younger patients, which seemed more apparent in men who were largely bothered by their symptoms at baseline (BII 7-13) or were less willing to spend the rest of their lives with the symptoms they had at baseline (IPSS-Q8 $\geq 4$ ).

Patients with a higher BII at baseline also have a higher IPSS since the two measures are correlated. Among men who received immediate intervention with FDC, the absolute change in IPSS increased with increasing BII at baseline. This effect, however, was not observed among men in the WW-no treatment subgroup. The larger relative improvement in IPSS for men who received FDC treatment and had a BII score of 7-13 at baseline is a novel and unique insight provided by this post hoc analysis. Although further data are needed, these findings suggest that rather than symptom severity at baseline, the degree of associated bother (as determined by BII) may be a more relevant indicator as to whether a patient will benefit from treatment or not.

After initial symptoms develop, many patients will postpone a visit to the physician and try to adjust their lifestyle to self-manage their symptoms, only seeking medical advice, when their LUTS eventually become too bothersome [9]. Ensuring men are aware of the importance of lifestyle advice in managing their symptoms is therefore an important focus, and communication plays an essential role. For example, doctors who are able to spend more time 
with their patients may be more successful in motivating them to adopt lifestyle changes. The extent to which the advice was incorporated or adhered to in the CONDUCT is unknown. Further analyses to determine whether the social, educational or professional status of a patient affected the adoption of lifestyle changes would be useful.

There are several limitations that should be considered when interpreting these results. Study-related limitations include the open-label design and the lack of a placebo arm. Limitations of these analyses include their post hoc nature and the absence of formal statistical testing. As the analyses described herein were not powered to detect differences, data were provided for descriptive purposes only and must be interpreted accordingly. Of note, statistical analyses of subgroups often result in the misinterpretation or misuse of clinical data. Since the original randomisation no longer applies, newly defined subgroups are likely to be biased; furthermore, when multiple subgroup analyses are performed, the probability of a false positive finding can be substantial [10].

In conclusion, some patients with moderately symptomatic BPH at risk of progression benefit from lifestyle advice alone. In this analysis, they are characterised by having a lower BII score at baseline; a novel finding that provides useful information for the care of patients and for future research. The CombAT study in men with moderate-to-severe symptoms showed symptomatic improvement with combination therapy, irrespective of BII [11]. Here, men with moderate symptoms but a higher bother score (7-13) appeared to have a substantial improvement in symptoms with combination therapy, even with lifestyle changes. The results of CONDUCT support guidelines that recommend conservative management for men with mild symptoms and those who are not bothered by moderate symptoms $[4,5]$ and suggest that using BII to further refine the selection of patients with moderate symptoms may provide a pathway to preventing the suboptimal management of $\mathrm{BPH}$.

Acknowledgments Medical writing support was provided by Spirit Medical Communications, funded by GlaxoSmithKline. The authors thank the following investigators and their patients for participating in the study. France: Abdel-Rahmène Azzouzi, Alain Boye, Christian Duroy, Daniel Bonneau, Gildas Ganuchaid, Gwendal Hulot De Collart, Jacques Tondut, Jean-Marc Aroun, Jérôme Basle, Jérôme Tondut, Loïc Boucher, Michel Lambert, Nicolas Tournemine, Patrick Muller, Philippe Igigabel, Pierre Leroy, Pierre André Ferrand, Robert Arnou, Thierry Schaupp. Germany: Christian Girke, Christian von Ostau, Detlef Quast, Hans-Carsten Braun, Joachim Dubiel, Joerg Willgerodt, Klaus Scheunpflug, Maren Schwickardi-Jerrentrup, Rainer Klammert, Ralk Eckert, Tilo Koettig, Wolf-Christian Hagel, Wolfgang Warnack. Greece: Anastasios Thanos, Andreas Skolarikos, Apostolos Apostolidis, Athanasios Papathanasiou, Georgios Moutzouris, Harilaos Katsifotis, Michael Melekos, Nikolaos Papandreou, Panagiotis Kalafitis, Pteros Perimenis. Italy: Cesare Selli, Francesco Montorsi, Giuseppe
Carrieri, Luigi Schips, Mauro Frongia, Paolo Gontero, Vincenzo Gentile, Vincenzo Mirone, Virgilio Cicalese. The Netherlands: Arnoldus G.H. Geboers, Hendrik A. Dirkse, Hendrikus G.M. Mevissen, Jos L. Bruins. Romania: Ioan Ioiart, Marius Dinu, Viorel Jinga. Spain: Javier Angulo Cuesa, Javier Extraniana, Javier Garcia Penit, Jose Martinez Javaloyas, José A. Gallego Sánchez, José María Del Rosal Samaniego, Maria Jose Requena Tapia, Manuel Fernández Arjona, Manuel Montesino. UK: Ramesh Corbarsanellore, Jonathan McFarlane, M. Naeem Akhtar, Neil Paul.

Authors' contribution C.G. Roehrborn was involved in protocol/ project development, data collection or management, data analysis and wrote/edited the manuscript; I. Oyarzabal Perez was involved in data collection or management, data analysis and wrote/edited the manuscript; E.P.M. Roos was involved in data collection or management, data analysis and wrote/edited the manuscript; N. Calomfirescu: was involved in data collection or management, data analysis and wrote/edited the manuscript; B. Brotherton was involved in protocol/ project development, data analysis and wrote/edited the manuscript; J.M. Palacios was involved in protocol/project development, data analysis and wrote/edited the manuscript; A. Vasylyev was involved in protocol/project development, data analysis and wrote/edited the manuscript; M.J. Manyak was involved in protocol/project development, data analysis and wrote/edited the manuscript.

\section{Compliance with ethical standards}

Conflict of interest C.G. Roehrborn has served as a consultant for GlaxoSmithKline. J.M. Palacios, A. Vasylyev and M.J. Manyak are all paid employees of GlaxoSmithKline and as such have historically received stock options. At the time of study, B. Brotherton was a paid employee of GlaxoSmithKline and is currently a paid employee of Parexel International. N. Calomfirescu's institution (Uroandromed) received monetary compensation from Pfizer, Lilly and Astellas for board membership and lectures; Pfizer, Sanofi and GlaxoSmithKline for consultancy work, and from Sanofi, GlaxoSmithKline, Storz and Angelini for lectures. I. Oyarzabal Perez and E.P.M. Roos have no conflict of interest to declare.

Ethical standard Written informed consent was obtained from each patient before the performance of any study-specific procedures. The study protocol was approved by a national, regional or investigational centre ethics committee or Institutional Review Board in accordance with International Conference on Harmonisation of Technical Requirements for Registration of Pharmaceuticals for Human UseGood Clinical Practice (ICH-GCP) and the ethical principles outlined in the Declaration of Helsinki 2008.

Open Access This article is distributed under the terms of the Creative Commons Attribution 4.0 International License (http://creativecommons.org/licenses/by/4.0/), which permits unrestricted use, distribution, and reproduction in any medium, provided you give appropriate credit to the original author(s) and the source, provide a link to the Creative Commons license, and indicate if changes were made.

\section{References}

1. Speakman M, Kirby R, Doyle S, Ioannou C (2015) Burden of male lower urinary tract symptoms (LUTS) suggestive of benign prostatic hyperplasia (BPH) - focus on the UK. BJU Int 115:508-519 
2. Parsons JK (2010) Benign prostatic hyperplasia and male lower urinary tract symptoms: epidemiology and risk factors. Curr Bladder Dysfunct Rep 5:212-218

3. Madersbacher S, Marszalek M, Lackner J, Berger P, Schatzl G (2007) The long-term outcome of medical therapy for BPH. Eur Urol 51:1522-1533

4. McVary KT, Roehrborn CG, Avins AL, Barry MJ, Bruskewitz RC, Donnell RF, Foster HE Jr, Gonzalez CM, Kaplan SA, Penson DF, Ulchaker JC, Wei JT (2011) Update on AUA guideline on the management of benign prostatic hyperplasia. J Urol 185:1793-1803

5. Oelke M, Bachmann A, Descazeaud A, Emberton M, Gravas S, Michel MC, Ndow J, Nordling J, de la Rosette JJ, European Associationof Urology (2013) EAU guidelines on the treatment and follow-up of non-neurogenic male lower urinary tract symptoms including benign prostatic obstruction. Eur Urol 64:118-140

6. Toren P, Margel D, Kulkarni G, Finelli A, Zlotta A, Fleshner N (2013) Effect of dutasteride on clinical progression of benign prostatic hyperplasia in asymptomatic men with enlarged prostate: a post hoc analysis of the REDUCE study. BMJ 346:F2109

7. Parsons JK, Schenk JM, Arnold KB, Messer K, Till C, Thompson IM, Kristal AR, Prostate Cancer Prevention Trial, Urologic Diseases in America Project (2012) Finasteride reduces the risk of incident clinical benign prostatic hyperplasia. Eur Urol 62:234-241

8. Roehrborn CG, Oyarzabal Perez I, Roos EP, Calomfirescu N, Brotherton B, Wang F, Palacios JM, Vasylyev A, Manyak MJ (2015) Efficacy and safety of a fixed-dose combination of dutasteride and tamsulosin treatment $\left(\right.$ Duodart $\left.{ }^{\circledR}\right)$ compared with watchful waiting with initiation of tamsulosin therapy if symptoms do not improve, both provided with lifestyle advice, in the management of treatment-naïve men with moderately symptomatic benign prostatic hyperplasia: 2-year CONDUCT study results. BJU Int 116:450-459

9. Scarpa RM (2001) Lower urinary tract symptoms: what are the implications for the patients? Eur Urol 40:12-20

10. Wang R, Lagakos SW, Ware JH, Hunter DJ, Drazen JM (2007) Statistics in medicine-reporting of subgroup analyses in clinical trials. N Engl J Med 357:2189-2194

11. Roehrborn CG, Barkin J, Siami P, Tubaro A, Wilson TH, Morrill BB, Gagnier RP (2011) Clinical outcomes after combined therapy with dutasteride plus tamsulosin or either monotherapy in men with benign prostatic hyperplasia (BPH) by baseline characteristics: 4-year results from the randomized, double-blind Combination of Avodart and Tamsulosin (CombAT) trial. BJU Int 107:946-954 